\title{
A ASSOCIAÇÃO DO VOCÁBULO ACCESS COM INTERNET NO REGISTRO ESCRITO DE DOIS GÊNEROS TEXTUAIS EM INGLÊS: UM ESTUDO À LUZ DA LINGUÍSTICA DE CORPUS
}

Jesiel Soares Silva

\section{RESUMO}

Este trabalho é o resultado de uma investigação empírica acerca da força de associação do termo access com internet entre um corpus de textos escritos formal e um informal. Usamos como medida de associação a Multual Information $\left(\mathrm{I}=\log _{2} \mathrm{O} / \mathrm{E}\right)$.). Como resultado, verificamos uma saliência quando o access é colocado com internet (5,13 e 31,19, respectivamente). Esta saliência apontou uma força de associação de 4,8 (formal) e 7,2 (informal).

PALAVRAS-CHAVE: linguística de corpus; força de associação; colocados.

\section{Considerações iniciais}

P ostular que a língua está em constante transformação é notadamente um lugar comum nos estudos acerca da linguagem. Ao longo dos anos, várias questôes de ordem social, econômica, geográfica, tecnológica têm promovido mudanças significativas na maneira em que a língua opera entre os atores que a realizam.

Um fator determinante nessas mudanças pode ser atribuído ao constante avanço tecnológico nos meios de comunicação, sobretudo após o surgimento da internet. Devido à abrangência e alternativas comunicativas da grande rede, muitas construçóes linguísticas em diversos idiomas têm sido cunhadas, 
adaptadas, relocadas. $\mathrm{Na}$ língua portuguesa, por exemplo, vários termos em inglês, advindos do mundo digital, já são recorrentes no uso diário da língua, bem como já estão incorporados nos dicionários, por exemplo: site, link.

Além de incorporar novos vocábulos à língua, percebemos que as novas tecnologias da informação e comunicação fazem com que termos tradicionais passem a adquirir novos significados e/ou significados mais abrangentes, por exemplo, os termos baixar e conectar, que, hoje em dia, além de seus significados tradicionais, também se referem a "fazer download" e "ter acesso à internet", respectivamente.

Para compreendermos essas transformaçóes de uma forma mais abrangente, é necessária uma investigação empírica da língua em uso, ou seja, um tipo de arcabouço teórico-metodológico capaz de gerar evidências acerca dessas transformaçôes, dos contextos, das evoluções temporais. Uma área dos estudos de linguagem capaz de tal feito é a Linguística de Corpus, que trabalha com a compilação de textos orais, escritos ou multimodais em diversos gêneros e, por meio de procedimentos estatísticos, de mensuração, consegue identificar alguns fatores, características, recorrências, padrôes da linguagem em uso.

Por intermédio da linguística de corpus, somos capazes de acessar e investigar a frequência das palavras, as maiores recorrências de alguns termos, bem como a associação de vocábulos com outros. Podemos ainda empreender um estudo em perspectiva diacrônica e entendermos o comportamento linguístico ao longo de um determinado espaço de tempo.

Pensando nessas questóes das transformaçôes da língua influenciadas pelo avanço tecnológico e fazendo uso da linguística de corpus como base teórica e metodológica, este artigo pretende fazer uma investigação empírica do vocábulo access da língua inglesa e buscar uma relação desse vocábulo com a palavra internet. Nossa hipótese é de que, devido às constantes transformações linguísticas subsidiadas pelos avanços tecnológicos, sobretudo a internet, o termo access, em gêneros textuais mais informais, tem sido associado fortemente ao termo internet de maneira mais contundente do que em gêneros mais formais.

A justificativa para a nossa hipótese reside no fato de que, por causa da entrada da internet em certa medida na vida cotidiana das pessoas em geral, a palavra access tem sido fortemente usada para se referir à internet mais do que para se referir a outros termos, principalmente em gêneros textuais mais informais, nos quais as mudanças notadamente ocorrem de forma mais rápida 
do que em gêneros mais formais.

Para tal, compilamos um corpus de textos escritos em língua inglesa com características mais informais e iremos compará-lo a um corpus de um gênero mais formal, buscando a força de associaçâo entre os dois termos mencionados. Assim, para os textos informais, construímos um corpus de aproximadamente 40000 palavras de essays (ensaios) em língua inglesa disponíveis na internet.

Esses ensaios têm características de textos mais informais por se tratarem da opiniāo de internautas sobre diversos temas (política, religião, educação, entre outros). Compararemos, então, esse corpus criado com o Corpus of Contemporary American English (COCA), na parte do gênero de escrita acadêmica, representando assim o que consideramos uma escrita mais formal.

O objetivo maior é investigar, por meio de um método estatístico, se o nível de associação do termo access com o colocado internet é mais significativo no corpus de textos mais informais do que no corpus de textos formais. Dessa forma, poderíamos fazer inferências acerca do processo de abrangência de significado do termo devido ao avanço da tecnologia, sobretudo da internet.

Este artigo está assim dividido: além das consideraçóes iniciais, faremos uma breve revisão teórica acerca da linguística de corpus, bem como seu lugar epistemológico nas teorias linguísticas. Em seguida, apresentaremos a metodologia do estudo, o percurso da criação do corpus, assim como as medidas estatísticas utilizadas. Por conseguinte apresentaremos a análise e discussão dos dados. Por fim apresentaremos as consideraçóes finais acerca do trabalho.

\section{Fundamentação teórica: bases epistemológicas, históricas e conceituais da Linguística de corpus}

A busca pela compreensão de algumas transformaçóes, alcances, abrangência, contextos da linguagem tem sido objeto de diversos estudos, correntes teóricas e escolas linguísticas ao longo das décadas. A complexidade dos fenômenos da linguagem vem sendo investigada sobre diversos aspectos, tanto por um ponto de vista mais estrutural e modelos teóricos, quanto por abordagens mais pragmáticas, que consideram a língua em uso. Seja na filosofia, na filosofia de linguagem ou nos estudos linguísticos, uma dualidade sempre recorrente e teoricamente antagônica é entre a visão racionalista e a visão empírica da linguagem. 
As raízes filosóficas para essa dualidade entre o racionalismo e o empirismo residem no século XVII na Europa, sobretudo entre René Descartes e seus seguidores, com o racionalismo, e alguns filósofos da escola inglesa, entre eles John Locke, com o empirismo. Para Descartes, o conhecimento provinha de ideias inatas e inerentes ao homem ao nascer. Reside neste pensamento o fato de que as ideias são universais e se diferenciam unicamente na adequação ao contexto. Um exemplo deste pensamento, para Descartes, seriam as ideias matemáticas (MORA, 1982) ${ }^{1}$.

Contrários a isso, alguns filósofos ingleses como Francis Bacon, David Hume e John Locke postulavam que só é possível conceber o conhecimento baseado nas impressóes sensitivas, ou seja, nas bases da sensibilidade dos nossos sentidos. Locke (1979) definiu a mente como um quadro em branco sobre o qual é gravado o conhecimento por intermédio das sensaçóes. Consequentemente, a sensação, a experiência, as tentativas e os erros culminam no conhecimento. Ao admitir que o conhecimento era proveniente das sensaçóes, Locke $(1979)^{2}$ refutou tanto a ideia de um conhecimento a priori quanto as ideias baseadas na abstração de conceitos não formulados pela sensibilidade e a experiência.

Nos estudos acerca da linguagem, a visão do inatismo cartesiano culminou na concepção racionalista de linguagem, cujo principal represente é Chomsky. Por outro lado, o empirismo dos pensadores ingleses foi a base epistemológica para a visão empírica da linguagem, cujo principal expoente é Halliday (BERBER SARDINHA, 2004) ${ }^{3}$.

Embasado no modelo gerativo-transformacional de concepção da linguagem, Chomsky $(1957)^{4}$ postulou que a língua deve ser compreendida no conjunto de possibilidades de sua realização vinculadas à competência dos falantes. Em outras palavras, na língua há possibilidades finitas de realização das estruturas e essas podem ser definidas e estabelecidas a priori.

Por outro lado, Halliday (1992) ${ }^{5}$ assevera que a linguagem é um siste-

1 MORA, J. F. A filosofia analítica: mudança de sentido em filosofia. Trad. Fernando Leorne. Rés: Porto, Portugal, 1982.

2 LOCKE, J. An essay concerning human understanding. New York: Oxford University, 1979.

3 BERBER SARDINHA, T Linguistica de Corpus. Barueri, SP: Manole, 2004.

4 CHOMSKY, N. Syntactic Structures. The Hague: Mouton \& Co., 1957.

5 HALLIDAY, M. A. K. Language as system and language as instance: the corpus as a theoreti- 
ma de probabilidades, portanto, alguns traços linguísticos são mais recorrentes que outros, a depender de determinados aspectos. Ou seja, mesmo que vários traços linguísticos sejam possíveis de ocorrer, eles não vão acontecer em frequências regulares ou equivalentes. Para Halliday (1992) a língua não deve ser analisada pelas possibilidades universais, mas pelas probabilidades contextuais, pois há uma correlação entre características linguísticas e situacionais (BIBER, 1988) ${ }^{6}$.

É importante ressaltarmos que a variação ocorrida na frequência dessas ocorrências não é aleatória, pois está condicionada a determinados aspectos como contexto de realização, associação entre termos, prosódia semântica, entre outros. A língua tem padrôes de realização e a compreensão desses padrôes é fundamental quando o que se busca é fazer inferências acerca dela (BERBER SARDINHA, 2004).

Baseados em Berber Sardinha (2004), podemos afirmar que, enquanto na visão racionalista o foco das investigações está na competência linguística e nos universais linguísticos, a linguística de corpus, amparada pela visão empirista, contempla o desempenho linguístico e a descrição linguística. Poderíamos sintetizar que o empreendimento da linguística de corpus consiste na investigação da linguagem por intermédio das evidências empíricas extraídas dela através da compilação de dados linguísticos (BERBER SARDINHA, 2004).

Desde que Sinclair $(1966)^{7}$ publicou Beginning the study of lexis, a Linguística de Corpus tem se ocupado em investigar a linguagem humana em uma perspectiva empírica. A partir de então, o campo de estudos com corpora tem se desenvolvido em vários países e o aparato tecnológico utilizado para os estudos tem obtido avanços consideráveis. Isso torna mais eficaz a compilação de corpora, bem como promove uma maior sofisticação no armazenamento, formatação e análise dos dados. Além do trabalho pioneiro de Sinclair (1966), vários outros autores posteriormente fomentaram as pesquisas e teorizaçóes

cal construct. In: SVARTIK, J. (org.) Directions in corpus linguistics. Proceedings of Nobel Symposium 82, Stockholm, 4-8 August 1991. Berlim/Nova York, De Gruyter, 1992. p. 61-78.

6 BIBER, D. Variation across speech and writing. Cambridge: Cambridge University Press, 1988.

7 SINCLAIR, J. McH. Beginning the study of lexis. In: BAZELL, C. E. In memory of R. Firth. Londres: Longman, 1966, p. 410-430. 
acerca dos estudos com corpora linguísticos (c.f BIBER, 1988; FRANCIS e KUCERA, 1982) ${ }^{8}$.

Berber Sardinha (2004, p. 18) considerou a definição de Sanchez (1995) ${ }^{9}$ interessante, pois nela está contida as bases e princípios da linguística de corpus: a origem, o propósito, a composição, a formatação, a representatividade, a extensão:

Um conjunto de dados linguísticos (pertencentes ao uso oral ou escrito da língua, ou a ambos), sistematizados segundo determinados critérios, suficientemente extensos em amplitude e profundidade, de maneira que sejam representativos da totalidade do uso linguístico ou de algum de seus âmbitos, dispostos de tal modo que possam ser processados por computador, com a finalidade de propiciar resultados vários e úteis para descrição e análise. (SANCHEZ, 1995, p. 8-9)

Uma característica importante da linguística de corpus é sua vinculação com o processamento computacional dos dados, pois a análise de corpora está sujeita à tecnologia dos computadores eletrônicos. Entretanto, para Berber Sardinha $(2000)^{10}$, antes mesmo da existência do computador já havia alguns trabalhos com característica de compilação de corpus - por exemplo, na Grécia antiga, quando Alexandre, o grande compilou o corpus helenístico, ou ainda na Idade Média com os grandes armazenamentos de citaçôes bíblicas.

Já na era dos computadores, o marco principal foi o Taggit, primeiro etiquetador morfossintático para computador, lançado em 1970 (BERBER SARDINHA, 2004) e desde então a instrumentação computacional tem se desenvolvido significativa e constantemente, facilitando o aprimoramento no tratamento com os dados de corpora.

8 FRANCIS, W. N.; KUCERA, H. Frequency analysis of English usage: lexicon and grammar. Boston: Houghton Mifflin, 1982.

9 SANCHEZ, A. Definición e historia de los corpos. In: SANCHEZ, A. et al. (orgs.). CUMBRE: corpus linguístico de español contemporaneo. Madri: SGEL, 1995, p. 7-24.

10 BERBER SARDINHA, T. Linguística de corpus: histórico e problemática. D.E.L.T.A. Vol. 16, N.o 2, 2000, p. 323-367. 
$\mathrm{Na}$ Linguística de Corpus, uma das formas de se investigar a recorrência de termos é através de seus colocados. Podemos analisar um termo (node ou nódulo) e o padrão que se forma em relação a seus colocados mais próximos. Assim, é possível, por intermédio de medidas estatísticas, mensurar o nível da força de associação de dois termos e delimitar se essa associação é aleatória ou se há alguma saliência estatisticamente significativa.

Os estudos sobre colocados têm sido foco de várias investigações da linguística de corpus e, para Berber Sardinha (2004), é o tipo de estudo com corpora mais recorrente na atualidade no meio científico. Podemos considerar que os estudos sobre colocados foram introduzidos por Firth, quando ele usou a célebre frase: "'Uma palavra deve ser julgada por sua companhia"11 (BERBER SARDINHA, 2004, p. 41).

Outros dois grandes pioneiros do trabalho com colocados foram Benson e colaboradores $(1986)^{12}$, que propuseram um dicionário de combinaçóes, e Kjellmer $(1994)^{13}$, que elaborou o primeiro dicionário de colocaçóes baseado em corpus (Dicionário BBI de colocaçóes). Este trabalho foi conduzido pela observação de padrôes recorrentes que foram identificados e medidos estatisticamente.

Levando em conta essa concepçóes e seguindo as definiçóes de Partington (1998, p. 16-17) $)^{14}$, nosso trabalho se encaixa no estudo colocacional de ordem estatistica, que averigua a probabilidade significativa de ocorrência de alguns colocados em relação a um node.

\section{Metodologia: A criação do corpus, o corpus de referência e a medida de associação}

Como mencionado na parte introdutória, pretendemos analisar o nível de associação do termo access com o colocado internet em dois contextos de

11 You shall judge a word by the company it keeps.

12 BENSON, M., BENSON, E., ILSON, R. (orgs.) The BBI dictionary of english word combinations. Amsterdã/Filadélfia: John Benjamins, 1986.

13 KJELLMER, G. A. A dictionary of English collocations: based on the Brown Corpus, v. 3. Oxford: Oxford University Press, 1994.

14 PARTINGTON, A. Patterns and meanings: using corpora for english language research and teaching. Amsterdã/Filadélfia: John Benjamnins, 1998. 
registro escrito da língua inglesa: um mais formal (escrita acadêmica) e o outro mais informal (ensaios livres).

Como registro formal, utilizaremos os dados do corpus de referência COCA, na parte de escrita acadêmica. Como registro informal, construímos um corpus pequeno de aproximadamente 40000 palavras contendo textos de caráter informal. Para fazer a comparação entre os dois corpora, utilizaremos a medida estatística de $M I$ (Multual Information), além da normalização dos dados para que haja a devida proporcionalidade.

Nesta parte metodológica, primeiro apresentaremos uma breve definição do COCA; em seguida, trataremos dos procedimentos de compilação do nosso corpus; por fim, apresentaremos a delimitação das medidas estatísticas nas quais nos apoiamos para a averiguação da força de associação entre os termos investigados.

\subsection{O COCA е о Free Essays}

O COCA (Corpus of Contemporary American English) é um corpus online ${ }^{15}$ de referência que tem armazenado textos desde 1990 até 2012 e continua crescendo. O COCA conta hoje com aproximadamente 450 milhóes de palavras e está vinculado à Brigham Young University. De acordo com o site do corpus, o COCA é o maior e mais balanceado corpus online com acesso livre.

Criado por Mark Davies, o COCA tem seus textos divididos em spoken, fiction, popular magazines, newspapers, e academictexts. Pelo COCA, é possível fazer comparaçóes entre gêneros textuais diversos (spoken, academic), bem como comparar as ocorrências com base na evolução do tempo (desde 1990).

Para fazer a comparação com os dados do gênero escrita acadêmica do COCA, fizemos a compilação de um pequeno corpus de aproximadamente 40000 palavras. Essas palavras provêm de textos de caráter informal, em forma de ensaio, dispostos no site www.123helpme.com.

Chamaremos esse corpus de Free Essays, visto que no site os internautas podem escrever textos sobre quaisquer temas e postar para que outros tenham acesso. Os textos avaliados, que seguem algum rigor e crivo, exigem que o internauta pague para ter acesso.

15 http://corpus.byu.edu/coca/ 
Entretanto, há um conjunto de textos que são de livre acesso e que não têm um processo de avaliação, por isso os consideramos mais informais. A partir destes textos gratuitos e informais, compilamos nosso corpus, o qual convertemos para o formato .txt, e utilizamos o software AntConc para efetuarmos o armazenamento e as análises dos dados.

No processo de compilação do Free Essays, seguimos as orientações de Berber Sardinha (2004, p. 19). Dessa forma, os textos são autênticos em linguagem natural, e o conteúdo do corpus foi escolhido criteriosamente levando em consideração o gênero escrito e o caráter informal.

Em relação ao tamanho do corpus, compilamos o maior número de palavras que conseguimos, pois a representatividade do corpus está diretamente ligada à sua extensão. Ainda seguindo as orientaçóes de Berber Sardinha (2004), em relação à tipologia, o Free Essays é um corpus escrito, contemporâneo, de amostragem, especializado e com a finalidade de estudo.

\subsection{Medidas de associaçâo: o MI Score}

Para Berber Sardinha (2004), há três modelos estatísticos para se calcular a força de associação entre termos lexicais. Primeiramente, a razão $O / E$ (observado/esperado), medida que exprime a probabilidade de vezes em que dois vocábulos podem ocorrer juntos dentro de um agrupamento limitado de palavras. Outra medida é o teste T ( $T$ score), que considera a posição dos itens analisados, ou seja, a palavra e seu colocado. Por fim, há uma medida de associação chamada de Informação Mútua (MI score), que utilizaremos neste trabalho.

O cálculo ${ }^{16}$ do $M I$ score também leva em consideração a razão logarítmica entre o que foi observado e o que é esperado (BERBER SARDINHA,

16 Matematicamente, podemos dizer que Multual Information é a mensuração da dependência inerente que pode ser explicitada em uma distribuição conjunta de $x$ e $y$ por si só em função da distribuição conjunta de $x$ e $y$ sob o pressuposto de independência. Partindo desse modelo matemático distributivo, Multual Information mensura o nível de dependência da seguinte maneira: I $(x, y)=0$ se, e somente se, $x$ e $y$ forem variáveis aleatórias independentes. Esse modelo pode ser expresso na equação:

$\log \left(\frac{p(x, y)}{p(x) p(y)}\right)=\log 1=0$. 
2004) e pode ser entendido na fórmula: $\mathrm{I}=\log _{2} \mathrm{O} / \mathrm{E}$. O objetivo principal em um exercício de medida de $M I$ score é verificar o quanto uma determinada frequência de um colocado em relação a um node é não-aleatória. Em outras palavras, quanto maior for o nível de não-randomização, teremos uma saliência estatisticamente importante no corpus em relação à linearidade esperada.

Posto isso, faremos a análise do termo access com o colocado internet levando em consideraçấo quatro colocados à direita e quatro colocados à esquerda. Optamos por esse número, uma vez que gostaríamos de analisar uma possibilidade maior de associação, posto que o colocado pode se referir ao node, mesmo estando a certa "distância" dele.

Para não incorrermos no risco de o colocado não fazer referência ao node, analisamos todas as ocorrência de access+internet (4e, 4d) no Free Essays, bem como analisamos algumas ocorrências do mesmo caso no COCA. Assim pudemos verificar que, em um espaço de quatro gaps à esquerda e quatro à direita, o colocado internet é referente ao node access (confira no Anexo).

\section{Análise e discussão dos dados}

Inicialmente, para estabelecer um aspecto geral da nossa investigação acerca da força de associação dos respectivos termos, fizemos uma análise da frequência de access no geral (access ${ }^{*}$ ), nos dois corpus analisados, bem como a frequência do termo dentro do gênero de escrita acadêmica do COCA, uma vez que esse gênero será nossa base de comparação com o Free Essays. O intuito maior foi termos uma ampla visão da ocorrência access, independente do colocado, em ambos os corpus:

Tabela 1 - Frequência bruta e normalizada de access no FreeEssays e no COCA

\begin{tabular}{|l|l|l|c|c|}
\hline Vocábulo & corpus & gênero & frequência & per mi \\
\hline access $^{*}$ & FreeEssays & ----- & 51 & 138.48 \\
\hline access $^{*}$ & COCA & Geral & 65168 & 140.44 \\
\hline access $^{*}$ & COCA & academic & 25259 & 277.37 \\
\hline
\end{tabular}

Como podemos observar na Tabela 1 , tanto no aspecto geral quanto na parte de escrita acadêmica (sobretudo na parte acadêmica) e, proporcionalmente considerando a normalização dos dados (per mi), a ocorrência de access 
é mais frequente no COCA do que no Free Essays. Uma primeira inferência que os dados nos apontam em relação ao esperado é a ideia de que, se há maior ocorrência do termo access no tanto no geral quando na parte acadêmica do $C O C A$, ao se analisarmos o mesmo termo com qualquer colocado, esperamos que essa análise aponte uma maior frequência de ocorrência do termo com o respectivo colocado no COCA, ao mesmo tempo, esperamos que ocorra uma menor frequência de ocorrência no Free Essays.

Assim, se no Free Essays a ocorrência de access com um determinado colocado for maior ou igual ao número de ocorrência no $C O C A$, teríamos uma saliência passível de ser investigada. Para averiguar melhor esta questáo, precisamos entender a frequência do termo access distribuída dentro dos gêneros do COCA:

Tabela 2 - Distribuição da frequência de access nos diversos gêneros do COCA

\begin{tabular}{|c|c|c|c|c|c|c|}
\cline { 2 - 7 } \multicolumn{1}{c|}{} & \multicolumn{6}{c|}{ GÊNEROS } \\
\hline & spoken & fiction & magazine & newspaper & academic & All \\
\hline Freq. & 7515 & 3391 & 16442 & 12561 & 25259 & 65168 \\
\hline Per mi & 78.63 & 37.50 & 172.06 & 136.96 & 277.37 & 140.44 \\
\hline
\end{tabular}

Como podemos notar na Tabela 2, a frequência da ocorrência de access na parte de escrita acadêmica do COCA é significativamente maior do que nos outros gêneros, sobretudo nos gêneros que consideramos mais informais, como o spoken e o magazine. A exceção apresentada pelos dados é em relação ao gênero fiction, que tem um número baixo de ocorrência comparado com os outros registros, sobretudo com o academic.

Ao compararmos os dois corpora de maneira proporcional (dados normalizados), podemos ver nas tabelas 1 e 2 que a frequência de access no academic do COCA (277.37) é significantemente mais expressiva que a frequência do mesmo termo no Free Essays (138.48).

Essa superioridade de frequência do respectivo termo no COCA (academic) em relação ao Free Essays nos faria pressupor que, na maioria dos casos, se access for exposto a algum colocado no COCA, a frequência de ocorrência com este colocado seria maior do que no Free Essays. Se acontecer o contrário, é porque há uma saliência importante que pode ser investigada; em outras palavras, haveria uma ocorrência que vai além do esperado. 
Primeiramente, se analisarmos os principais colocados de access (4e, 4d), o principal colocado de access no academic do COCA é information; enquanto que no Free Essays é internet. Temos assim o primeiro indício de que access tem uma força de associação maior com internet no Free Essays do que no academic do COCA. Entretanto, essa ainda não é uma medida estatística. Posto isso, a seguir apresentaremos a frequência de access com o colocado internet distribuída nos gêneros do COCA:

Tabela 3 - Distribuição da frequência de accesscom internet nos diversos gêneros do COCA

\begin{tabular}{|c|c|c|c|c|c|c|}
\cline { 2 - 7 } \multicolumn{1}{c|}{} & \multicolumn{6}{c|}{ GÊNEROS } \\
\hline & spoken & fiction & magazine & newspaper & academic & all \\
\hline Freq. & 219 & 39 & 653 & 583 & 467 & 1961 \\
\hline Per mi & 2.29 & 0.43 & 6.83 & 6.36 & 5.13 & 4.23 \\
\hline
\end{tabular}

Se compararmos as tabela 2 e 3 perceberemos algo importante. Quando se trata frequência de access no geral, com os mais diversos tipos de colocados, o academic do COCA aparece em destaque com um número significativamente maior do que os outros gêneros. Entretanto, quando analisamos o vocábulo access com o colocado internet, percebemos que a frequência no academic é menor do que em gêneros mais informais como magazine. Dessa forma, podemos inferir que no academic do COCA há muita frequência de access com diversos colocados, mas, quando se trata do colocado internet, a frequência é menor do que nos gêneros mais informais.

Essa inferência nos leva ao ponto chave desta pesquisa: a comparação entre a força de associação de access com internet nos dois corpora para, através da media estatística do MI score, determinarmos se a diferença entre os dois casos tem alguma significância estatística ou se apenas é uma aleatoriedade: 
Tabela 4 - comparação entre a associação de access com internet no FreeEssays e no COCA

\begin{tabular}{|c|c|c|c|c|c|}
\cline { 2 - 6 } \multicolumn{1}{c|}{} & \multicolumn{2}{c|}{ Ocorrênciade access $^{*}$ no geral } & \multicolumn{3}{c|}{ Ocorrênciade access ${ }^{*}$ com internet } \\
\hline COCA & frequência & per mi & frequência & per $m i$ & MI score \\
\cline { 2 - 6 } (academic) & $\begin{array}{c}7236 \\
(0.06 \%)\end{array}$ & 18.8 & $\begin{array}{c}467 \\
(6.43 \%)\end{array}$ & 5.13 & 4.8 \\
& frequência & permi & frequência & per mi & MI score \\
\cline { 2 - 6 } FreeEssays & $\begin{array}{c}51 \\
(0.12 \%)\end{array}$ & 12.6 & $\begin{array}{c}17 \\
(33.3 \%)\end{array}$ & 31.19 & 7.2 \\
\hline
\end{tabular}

Pela Tabela 4, notamos que o termo access tem uma maior força de associação com internet nas Free Essays (7.2) do que no acadêmico do COCA (4.8). Sendo assim, temos evidências estatísticas para afirmar que, nos casos estudados, a força de associação de access com internet é maior em textos informais do que formais. Para confirmar essa força de associação apresentada nos dados, no quadro a seguir são apresentados os resultados obtidos no AntConc acerca dos colocados de access ordenados pela medida de MI Score.

Podemos perceber neste ranking que vários colocados têm um valor de MI score próximo do valor obtido com internet. Entretanto, se olharmos a frequência de ocorrência dos ermos, veremos que o número de internet é consideravelmente maior.

Figura 1 - Ranking dos colocados de access considerando o MI

\begin{tabular}{|c|c|c|c|c|c|c|c|c|}
\hline \multicolumn{2}{|c|}{ Concordance } & \multicolumn{3}{|c|}{ Concordance Plot } & File View & \multicolumn{2}{|c|}{ Clusters } & Collocates \\
\hline \multicolumn{9}{|c|}{ Total No. of Collocate Types: 228 Total No. of Collocate Tokens: 522} \\
\hline Rank & \multicolumn{2}{|c|}{ Freq } & Freq(L) & Freq(R) & \multicolumn{2}{|l|}{ Stat } & \multicolumn{2}{|c|}{ Collocate } \\
\hline 49 & \multicolumn{2}{|l|}{1} & 0 & 1 & \multicolumn{2}{|l|}{7.33775} & \multicolumn{2}{|c|}{ technical } \\
\hline 50 & \multicolumn{2}{|l|}{1} & 0 & 1 & \multicolumn{2}{|l|}{7.33775} & \multicolumn{2}{|c|}{ abilities } \\
\hline 51 & \multicolumn{2}{|l|}{1} & 0 & 1 & \multicolumn{2}{|l|}{7.33775} & \multicolumn{2}{|c|}{ diverse } \\
\hline 52 & \multicolumn{2}{|l|}{1} & 1 & 0 & \multicolumn{2}{|l|}{7.33775} & \multicolumn{2}{|c|}{ telephone } \\
\hline 53 & \multicolumn{2}{|l|}{1} & 0 & 1 & 7.33775 & & actu & aly \\
\hline 54 & 1 & & $\mid 1$ & 0 & 7.33775 & & stop & \\
\hline 55 & 4 & & 4 & 0 & 7.26736 & & havi & \\
\hline 56 & 17 & & 14 & 3 & 7.23934 & & inte & met \\
\hline 57 & 1 & & $\mid 1$ & 0 & 7.07471 & & adva & aced \\
\hline 58 & 1 & & 1 & 0 & 7.07471 & & elem & entary \\
\hline 59 & 1 & & 1 & 0 & 7.07471 & & netw & prying \\
\hline 60 & 1 & & | 0 & 1 & 7.07471 & & purp & bses \\
\hline 61 & 1 & & 1 & 0 & 7.07471 & & moni & Eor \\
\hline 62 & 1 & & |lo & 1 & 7.07471 & & lect & urers \\
\hline 63 & 1 & & $\mid 1$ & 0 & 7.07471 & & cont & $\operatorname{col}$ \\
\hline$<$ & $<$ & $\geqslant$ & $\mid<$ & $<$ & $<$ & $>$ & $\mid<<$ & \\
\hline
\end{tabular}


Vemos, por exemplo, que o colocado advanced tem uma força de associação de 7.07, mas houve apenas uma ocorrência no corpus; em outras palavras, advanced ocorreu apenas uma vez no corpus e quando ocorreu foi como colocado de access. Por outro lado, no caso de internet, há um maior número de ocorrências e ainda assim o MI score é 7.2, um valor significativo que corrobora com a nossa hipótese inicial.

\title{
5. Considerações finais
}

Retomando a ideia inicial, este trabalho mostrou um pequeno indício de que a evolução tecnológica contribui para algumas mudanças na língua, seja na criação de novos termos, seja na abrangência de significaçáo de outros. Além disso, pudemos notar que, no gênero escrito, essas mudanças ocorrem primeiramente em textos notadamente mais informais.

Como limitação deste trabalho, temos o fato de que o corpus construído foi pequeno; mesmo que tenhamos normalizado os dados, tivemos apenas uma nuance da representação que pretendíamos. Sugerimos, para estudos futuros que abordem a mesma questão, que sejam feitos levantamentos históricos em corpus que tenham essa característica para assim se conhecer a evolução dos termos e seus colocados ao longo dos anos. Sobretudo, neste aspecto do termo access, seria válido fazer um levantamento de seus colocados antes e depois do surgimento da internet.

O constante avanço dos aparatos tecnológicos favorece a Linguística de corpus nesse aspecto, por isso acreditamos que investigar a língua em seu caráter probabilístico, buscando padrōes e fazendo inferências, é uma agenda importante nos estudos acerca da linguagem humana.

\section{THE ASSOCIATION OF THE WORD 'ACCESS' WITH 'INTERNET' IN THE TWO WRIT'TEN TEXT GENRES IN ENGLISH: A STUDY ON OF CORPUS LINGUISTICS}

\author{
ABSTRACT: \\ This paper is the result of an empirical research on the \\ association between the word 'access' with 'internet' in \\ two corpora of written text: formal and informal. As
}


an association measure we made use of Multual Information Score $\left(\mathrm{I}=\log _{2} \mathrm{O} / \mathrm{E}\right)$.). As a result, we verified a highlight when 'access' is displayed with 'internet' $(5,13$ e 31,19 , respectively). This fact has guided us to an association of 4,8 (formal) and 7,2 (informal)

KEYWORDS: Corpus Linguistics; Association; Collocates

Recebido em: 21/01/2013

Aprovado em: 12/08/2013

\begin{tabular}{|c|c|c|}
\hline \multicolumn{3}{|c|}{ ANEXO } \\
\hline $\begin{array}{r}\text { Essentially, this expansion along with } \\
\text { Internet }\end{array}$ & Access & $\begin{array}{l}\text { created a new way to access and send } \\
\text { information }\end{array}$ \\
\hline Georgia's K12 school districts have Internet & Access & $\begin{array}{l}\text { and use a variety of on-line educational } \\
\text { software }\end{array}$ \\
\hline Internet in its simplest form enables one to & Access & emails; this too is a source of information \\
\hline a cell phone & Access & ernet which allows students \\
\hline solutions to stop students from gaining & Access & to the internet have been found to \\
\hline money and we can still monitor your Internet & Access & Also for disciplinary purposes if you fail \\
\hline century learning work for us. Internet & Access & and more constructivist teaching practices \\
\hline $\begin{array}{r}\text { objective was to see if well-supported } \\
\text { Internet }\end{array}$ & Access & cha \\
\hline was observed had a level of Internet & Access & technical support and staff development \\
\hline Thus, the impact of classroom Internet & Access & $\begin{array}{l}\text { could be examined in an environment where } \\
\text { the typical }\end{array}$ \\
\hline In 1996 schools brought Internet & Access & $\begin{array}{l}\text { into the classroom, allowing students and } \\
\text { teachers }\end{array}$ \\
\hline $\begin{array}{r}\text { Yet another benefit having computers and } \\
\text { internet }\end{array}$ & Access & $\begin{array}{l}\text { in the classroom is the ability of students to } \\
\text { be able }\end{array}$ \\
\hline It can be quite a liability to have internet & Access & $\begin{array}{l}\text { in the classroom; teachers must be sure they } \\
\text { protect }\end{array}$ \\
\hline $\begin{array}{r}\text { In 2001, almost all public schools with } \\
\text { internet }\end{array}$ & Access & $\begin{array}{l}\text { (96 percent) used various technologies or } \\
\text { procedures }\end{array}$ \\
\hline technologies or procedures to control internet & Access & to inappropriate material on the internet \\
\hline $\begin{array}{r}\text { the prohibitively high cost of telephone and } \\
\text { Internet }\end{array}$ & Access & $\begin{array}{l}\text { charges. Educators also face the challenge of } \\
\text { designing }\end{array}$ \\
\hline to an infinite amount of information with & Access & $\begin{array}{l}\text { to the Internet. With so much information } \\
\text { available }\end{array}$ \\
\hline
\end{tabular}




\section{Exemplos de Ocorrência do node access colocado com internet (4d, 4e) no corpus COCA}

of the file system of the CRIS UNS server. The folder is not directly accessible through the Internet, but digital contents can be downloaded using a Java servlet. school oersonnel can use the computers for grading, writing papers or assignments, and accessing information on the Internet. \# The implications for parents are also impc provide schools with a very convenient bad-weather backup -. as long as students' Internet access spreads as quickly. \# Source: Chinese Web media company Sina, May 1

1. As having a family decreases in importance in the next 30 years, access to the Internet will increase, suggesting that the impulse for human contact will take and (d) share updates on action plan items. Smart phones with Internet access also are convenient ways to transmit information and stay connected. Because all participan general. Shelburne noted problems with Digital Rights Management (DRM) and with Internet access along with other technical difficulties such as the need for special reader , the e-books discussed are aggregations of titles purchased from vendors in package deals and accessible over the Internet. \# What few articles do discuss e-readers in a , responsible citizen participation, contributed articles challenge. ICTs, including cellphones and Internet access, serve as technological mediation between political administr the world and said, " Here, fly into the world of information, access the Internet and the worldwide Web, " they would reply, "I'm

to in the first place. But if a government were to shut down Internet access or ban cell phones, it would risk radicalizing otherwise pro-regime citizens or harming the

\# Most notably, Clinton announced funding for the development of tools designed to reopen access to the Internet in countries that restrict it. This " instrumental " approach of its operations under the federal E-Rate program, which helps fund telephone and Internet access for schools and libraries. The provider was SBC Communications Inc, $z$ of websites by lawyers. "A lawyer website can provide to anyone with Internet access a wide array of information about the law, legal institutions and the value of the stakes are even higher, \#GENERAL TRENDS Despite the fact that their Internet access is free of outside manipulation, most Palestinian activists do not reveal their narr of Statistics estimates that in 2009, 28.5 per cent of Palestinian households had Internet access2 though these statistics do not account for the widespread use of hundreds the majority of the Arab world as Israel provides the Palestinian territories with unfettered Internet access.4 \# FDD instructed ConStrat not to provide percentages for the s hybrid learning will find a way to help bridge the gap for learners without Internet access. \# THE LIBRARY IN THE CENTER Our blended online and face-to-face program thr! transgender people often comes through the media. Today, the internet is providing greater access to information and people are gaining this awareness at a younger age, . 2004; 94(12):2050. \#64, Gilmour JA, Reducing disparities in the access and use of Internet health information, a discussion paper. Int J Nurs Stud.

methods to recruit panelists. To ensure participation of low-income individuals and those without Internet access, KN provides access to the Internet and hardware if need - To ensure participation of low-income individuals and those without Internet access, KN provides access to the Internet and hardware if needed. 3940 \# We invited a ral as heightened concern for the environment (Bhattarai and Hammig 2004). Increased Internet access (Godfray 2007) and more published field guides (Pearson and Shetterl \# \# It was 1994, Newly devised graphical Web interfaces made the Internet widely accessible. Scanning technology was just becoming commercially avalable, In a bold $\mathrm{mi}$ their growing presence include concerns about the environmental and other consequences of neoliberal globalization, access to the Internet, and the emergence of relativel hampers the freedoms of the print and broadcast media, Malaysians enjoy widespread and uncensored access to the Internet, in large part because the government is eagr 